\title{
Entre médicos y sanadores: gestionando sentidos y prácticas sobre el proceso de salud-enfermedad- atención en un movimiento carismático católico argentino
}

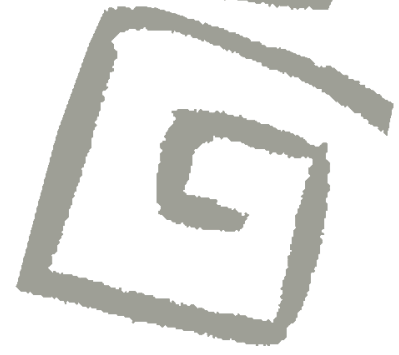

\author{
Between doctors and healers: managing meanings \\ and practices of the health-disease-care process in an \\ Argentine Catholic charismatic movement
}

Ana Lucía Olmos Álvarez

${ }^{1}$ Doctora en Antropología Social. Becaria posdoctoral, Consejo Nacional de Investigaciones Científicas y Técnicas, con sede en el Instituto de Altos Estudios Sociales, Universidad Nacional de San Martín, Buenos Aires, Argentina. $\triangle$ iD
RESUMEN Este trabajo describe y analiza las trayectorias y las estrategias de búsqueda de sanación entre los miembros de un movimiento católico carismático de sanación en Argentina. A partir de una investigación etnográfica realizada entre julio de 2009 y abril de 2015, se focaliza en las concepciones, prácticas y experiencias en torno a la salud-enfermedad-atención. Se señala que el evento de enfermedad requiere una respuesta cognitiva y otra práctica para su gestión e involucra, principalmente, dos recursos terapéuticos: la biomedicina y las terapias religiosas. Asimismo, se postula que cada recurso es referido en momentos específicos de las trayectorias y el pasaje de uno a otro implica el cambio de una noción restringida por una holística del proceso salud-enfermedad-atención.

PALABRAS CLAVES Religión; Proceso Salud-Enfermedad; Etnografía; Curación por la Fe; Terapias Espirituales; Catolicismo; Argentina.

ABSTRACT This work describes and analyzes the trajectories and strategies in the search for healing among participants of a charismatic Catholic healing movement in Argentina. Using ethnographic research carried out between July 2009 and April 2015, the article focuses on conceptions, practices and experiences surrounding health-disease-care processes. It is highlighted that disease management requires both a cognitive and a practical response, in this case involving two primary therapeutic resources: biomedicine and religious therapies. It is also postulated that each resource is sought at specific moments in the trajectories and the passage from one to another implies a change from a restricted to a holistic notion of the health-disease-care process.

KEY WORDS Religion; Health-Disease Process; Ethnography; Faith Healing; Spiritual Therapies; Catholicism; Argentina. 


\section{INTRODUCCIÓN}

La creciente visibilidad adquirida por grupos y sanadores a quienes se les atribuye efectos taumatúrgicos sobre la vida cotidiana de los actores activó aquel interrogante de antropólogos como Evans-Pritchard ${ }^{(1)}$, Lévi-Strauss ${ }^{(2)}$ y Turner ${ }^{(3)}$, entre otros, acerca de la eficacia simbólico-práctica de ciertas acciones sobre el orden social. En los últimos años, numerosos cientistas sociales centraron sus esfuerzos en la comprensión de las múltiples vinculaciones entre la salud y las terapéuticas religiosas ${ }^{(4,5,6,7,8,9,10)}$. Estos lazos, interpretados como signos de transformación del paisaje religioso, insertaron a la vez el binomio saludreligión en una discusión mayor referida al lugar de las últimas en las sociedades contemporáneas ${ }^{(11,12,13)}$. En este contexto, la vía elegida para explorar estas intersecciones es el análisis de las trayectorias y las estrategias de búsqueda de sanación que los actores despliegan en el terreno socioreligioso argentino (específicamente, aquellas contenidas dentro del ámbito católico carismático), focalizando en las concepciones, prácticas y experiencias en torno a la salud-enfermedad-atención.

El interés por alcanzar estos objetivos surge de algunos emergentes y hallazgos de la investigación etnográfica efectuada para mi tesis doctoral en Antropología Social Construyendo carismas en el catolicismo contemporáneo argentino. Un estudio etnográfico sobre el movimiento en torno al Padre Ignacio Peries ${ }^{(14)}$. La investigación fue realizada entre los seguidores de "un cura sanador" (a lo largo del escrito usaré las comillas dobles para señalar las categorías de los actores) y abordó el proceso de construcción simbólica de la figura de un sacerdote católico investido socialmente de competencias, habilidades y rasgos valorados como excepcionales y al movimiento carismático de sanación formado en torno a su figura. El principal aporte de ese trabajo fue la comprensión del proceso de configuración de la "persona carismática" como resultado de las relaciones sociales que ciertos actores entablan con él, que influyen sobre la producción de significados y generan tensiones con el medio institucional del cual emerge. En el presente trabajo, me centraré en el área de incumbencia específica que los seguidores asignan al carisma de Peries: la salud y la enfermedad.

A lo largo del artículo señalo que el evento de enfermedad requiere una doble respuesta para gestionarlo: una cognitiva, que implica la interpretación y la explicación, y otra práctica, que involucra las acciones para su resolución. En este marco, el caso que presento reviste características particulares respecto de dicha gestión, ya que resulta de una configuración singular entre religión y salud. Veremos que en las concepciones, prácticas y experiencias del proceso salud-enfermedad-atención convergen distintos recursos para significar y actuar sobre los eventos de enfermedad: uno, que abreva en el campo biomédico y, otro, en el religioso. Postulo también que cada recurso es referido en momentos específicos de las trayectorias y que el pasaje de uno a otro conlleva una modificación en la conceptualización de este proceso.

El trabajo propone un recorrido en cuatro secciones. La primera muestra algunos abordajes del proceso salud-enfermedad-atención desde las ciencias sociales. Luego, la segunda se centra en la presentación del caso y la metodología empleada para la construcción y análisis de los datos empíricos. Finalmente, la tercera y cuarta describen y analizan las respuestas cognitivas y prácticas, considerando los dos recursos señalados respectivamente. Por estos medios, busco dar cuenta de una de las modalidades en que los actores configuran la gestión simbólico práctica de la salud, la enfermedad y el sufrimiento.

\section{Salud-enfermedad-atención: una mirada desde las ciencias sociales}

El proceso de la enfermedad comienza con la autopercepción de algún cambio en la experiencia corporal y anímica que irrumpe en el devenir cotidiano de los actores. Estos pondrán en juego explicaciones y posibles soluciones, culturalmente informadas, a dicha 
interrupción $^{(15)}$. Para nombrarlas, en tanto conjunto de representaciones y prácticas socialmente construidas sobre la salud-enfermedad-atención, desde las ciencias sociales se ideó el término "sistemas médicos". Estos engloban representaciones; dinámicas de roles entre curadores de distinta índole (biomédicos, sanadores o chamanes) y pacientes; y jerarquías, competencias y cooperación de saberes $^{(16)}$. Conjugando estos lineamientos ${ }^{(10,15,16)}$ entenderé aquí que el proceso salud-enfermedad-atención es de naturaleza social y se inserta en un entramado de relaciones personales e institucionales que lo constituyen y limitan. En consecuencia, enfermar está lejos de ser un episodio individual, sino que es un evento en torno al cual giran prácticas, símbolos y discursos colectivos para interpretar y actuar sobre los daños a la salud, el sufrimiento y la desgracia.

Diversas investigaciones para contextos socioculturales disímiles comprobaron dos fenómenos simultáneos y entrelazados: la pluralidad de sistemas médicos y los procesos de selección y combinación por parte de los actores ${ }^{(9,10,15,16,17,18,19,20,21)}$. El primero de estos fenómenos no está exento de jerarquías ya que los procesos económico-políticos y técnico-científicos instituyeron a determinados sistemas como hegemónicos. A la vez, produjo curadores socialmente reconocidos y autorizados para atender un rango determinado de daños a la salud individual y colectiva $^{(22)}$. La biomedicina (nombre con el que refiero a la medicina occidental científica, oficialmente reconocida y ofertada en hospitales y centros de salud públicos y privados) constituye una de las formas de atender el proceso salud-enfermedad. Ciertos autores, la identifican como la más correcta y eficaz en un gran número de sociedades ${ }^{(22)}$; mientras que otros señalan que la idea natural de la enfermedad sostenida por los biomédicos no es la más común entre los grupos ni estaría generalizada en el mundo occidental ${ }^{(16)}$. En este sentido, ya en 1978, Kleinman advertía que "entre el $70 \%$ y el $90 \%$ de todos los episodios autoreconocidos de enfermedad se manejan exclusivamente fuera del sistema formal de atención de salud"(21). A pesar de estos señalamientos, numerosos trabajos realizados en diversos escenarios ilustran las formas en que la biomedicina modela la interpretación que los individuos hacen de los eventos que conciernen a su cuerpo y de su experiencia íntima, por medio de la creación de un consenso naturalizado y percibido como espontáneo ${ }^{(10,17)}$. A lo largo del trabajo, señalaré las modalidades en que el discurso biomédico atraviesa y constituye las experiencias y discursos de los seguidores/enfermos y del movimiento religioso en cuestión.

Otro fenómeno constatable en contextos socioculturales disímiles es la articulación de sistemas médicos. Frente a esto, diversos cientistas sociales se concentraron en clasificar las medicinas y terapias que son objeto de selección y articulación $n^{(9,18,21)}$. Estas sistematizaciones, al buscar trascender el biomédicocentrismo, toman en cuenta la interacción de los actores con diversos tipos de terapias y el contexto social en que la enfermedad es percibida y tratada. Además de la biomedicina, identifican las medicinas tradicionales (curanderismo y shamanismo), religiosas (practicadas en el contexto de las creencias y rituales de grupos religiosos), alternativas/ complementarias (que comprenden las terapias ligadas al new age), y el autotratamiento. A pesar de sus especificidades, comparten el abordaje holístico de la salud ${ }^{(9)}$ y las terapias suponen la sociabilidad para su legitimidad y eficacia simbólico práctica ${ }^{(23)}$.

Al circunscribirnos al espectro de las terapias religiosas, la curación -tal como se desarrolla en comunidades y grupos carismáticos- motivó un caudal de estudios en el contexto latinoamericano sobre sus dimensiones rituales y su impacto en el plano institucional. Respecto a los primeros, encontramos trabajos focalizados en los contenidos extáticos (cantos, danzas y glosolalia) $)^{(24)}$, en su proximidad con las prácticas shamánicas ${ }^{(25)}$ y con otras denominaciones religiosas $^{(26)}$, y acerca del universo simbólico que promueven ${ }^{(27,28,29,30)}$. Al centrarse en las vivencias inmediatas y emocionales de la presencia de Dios y enfatizar el contacto directo con la divinidad y los carismas bíblicos, las prácticas carismáticas impulsan 
modelos rituales, doctrinales y organizacionales que resultan novedosos en el escenario católico. Por ello, la expansión e impacto de estas innovaciones fueron analizados en relación con las modificaciones de la sociedad moderna en general ${ }^{(31,32)}$, como respuesta de la iglesia católica ante la pérdida de la hegemonía que la caracterizó otrora ${ }^{(33,34)}$ y en tanto lugar de paso hacia religiosidades más individualistas y reflexivas ${ }^{(29)}$.

Me interesa aquí indagar en las maneras en que estos fenómenos identificados a nivel macro se expresan en las biografías de los actores. Para ello recurro al concepto medically pluralistic setting ${ }^{(15)}$, el cual toma como punto de partida un escenario nutrido por diversas tradiciones curativas, en el que los recursos del conocimiento cultural (en tanto entendimientos culturalmente disponibles) son utilizados situacionalmente para otorgar las respuestas cognitivas y prácticas que los eventos de enfermedad requieren. A lo largo de este artículo y dando una lectura renovada y más profunda a mis datos ${ }^{(5)}$, entenderé la cosmología religiosa como un recurso terapéutico sui generis que junto a otras prácticas y saberes curativos son adoptados por los actores.

Siguiendo estos lineamentos y con base en mi experiencia etnográfica, indagaré entonces la configuración médicamente plural, tal como se desarrolla entre los pacientes del cura Peries, caso que presento a continuación.

\section{Etnografiando la gestión de la salud- enfermedad-atención}

Ignacio Peries nació en Sri Lanka en el seno de una familia católica en 1950, se ordenó sacerdote en Inglaterra y está radicado en la ciudad de Rosario (Santa Fe, Argentina) hace más de 35 años. Allí es párroco de la Natividad del Señor, parroquia situada en el distrito noroeste rosarino y titular de la asociación clerical Cruzada del Espíritu Santo, la cual fue fundada en la década de 1960 bajo el influjo del Concilio Vaticano II (19621965). Su figura es altamente visible por diversas razones enlazadas, entre ellas, su capacidad sanadora ${ }^{(5,35),}$ la acción misionera y social que desarrolla ${ }^{(36)}$ y los vínculos construidos con figuras políticas a lo largo de los $\operatorname{años}^{(36,37)}$. Estas características lo posicionan socialmente como líder de un movimiento carismático de sanación.

Me centraré en el primer motivo ya que es la piedra basal de su popularidad sobre la que se encaraman los otros dos elementos. La capacidad sanadora de Peries es difundida por distintas narrativas (comentarios, testimonios y rumores) que circulan tanto en las interacciones cara a cara como por los medios de comunicación y las redes sociales. En ellas, el sacerdote es percibido como "sanador" y especialista en la resolución de desórdenes emocionales, problemas de fertilidad y "enfermedades mortales" como cáncer. La sanación se realiza por medio de la "imposición de manos" y las "bendiciones", las cuales constituyen una experiencia religiosa central como bien buscado a partir de la confianza en que la intervención numinosa tendrá consecuencias reales en la vida de sus seguidores.

La caracterización de este movimiento como carismático responde más a la centralidad de la sanación, del contacto físico (entre fieles y celebrante) y de la efervescencia emocional en el desarrollo de las liturgias, que a una relación orgánica con el homónimo Movimiento de la Renovación Carismática Católica en Argentina. Es decir, responde a un criterio simbólico ritual antes que institucional. Asimismo, lo refiero como movimiento por dos razones principales. Primero, porque si el término designa un esfuerzo colectivo por modificar los modos instituidos de hacer de una sociedad ${ }^{(38)}$, este caso exhibe características novedosas respecto de los modos de gestión de la salud-enfermedad. Segundo, la participación de algunos de sus seguidores es coyuntural y responde a la percepción de una problemática a la cual la propuesta del sacerdote puede resolver, generando en consecuencia formas alternativas de participación en las comunidades religiosas. Ahora bien, los cambios en los modos de hacer como la dinámica coyuntural, en tanto punto de partida de pertenencias móviles, tienen lugar dentro de un marco religioso institucional 
que define características y deberes, a la vez que establece límites y condiciones a los actores involucrados en dichos procesos. En este sentido, encontramos dos ordenamientos que operan superpuestos: por un lado, aquel que responde a la institución eclesiástica y, por el otro, el de la asociación clerical dirigida por el sacerdote ceilandés. Peries representa al primero, ya que es miembro de la jerarquía clerical y, dados sus rasgos individuales y los socialmente atribuidos, constituye una figura incómoda, y tanto su accionar como los vínculos forjados con otras esferas sociales no están exentas de $\operatorname{conflictos}^{(36)}$. El segundo ordenamiento es aquel que estructura internamente a la Cruzada del Espíritu Santo, articula diversos dispositivos que permiten su anclaje territorial circunscripto al distrito noroeste rosarino y organiza el trabajo del numeroso grupo de colaboradores que integran la parroquia y tienen, como veremos, un rol capital durante las bendiciones. En suma, es la puja entre la rigidez de los marcos institucionales y la flexibilidad de las prácticas de los adherentes la que otorga parte de su impronta al caso que presento.

\section{ASPECTOS METODOLÓGICOS}

Llevar adelante una investigación acerca de un fenómeno masivo y en constante expansión como es el movimiento en torno a Peries requirió de una estrategia metodológica que diera cuenta de la pluralidad de tramas de sentidos superpuestas que se muestran y opacan en la dinámica social(39). Por ello, al construir mi abordaje de este particular movimiento elegí la aproximación etnográfica que permite un acercamiento a los procesos sociales desde la perspectiva de sus miembros ${ }^{(40)}$ y conocerlos en el contexto de su experiencia y complejidad cultural. Pude así comprender las maneras en que los actores interpretaban sus experiencias y las de terceros, las apreciaciones sobre la realidad y sobre ciertos eventos evaluados como problemas o como sus posibles soluciones y atender, en particular, las acciones, las emociones y las historias de los devotos en su acercamiento al cura. La investigación abarcó desde julio de 2009 a abril de 2015. Durante el trabajo de campo, realicé observación participante de numerosas misas desplegadas por Peries, principalmente, y por otros sacerdotes de la Cruzada del Espíritu Santo: las celebradas los días de semana y los fines de semanas, la de las mujeres, la de los jóvenes, como también de bendiciones grupales. Presencié, además, eventos centrales del calendario litúrgico católico como el Viacrucis de la Pasión de Cristo, el día de la Natividad de la Virgen María y el festejo de Pentecostés. Asimismo, participé en viajes organizados desde distintos puntos del país para asistir a las ceremonias en Rosario y relevar así los flujos de concurrencia religiosa y las diferentes redes locales de adherentes como también forjar contactos para futuras entrevistas con asistentes.

Un contrapunto indispensable a estas observaciones y participaciones lo constituyeron el análisis de fuentes y las entrevistas. El corpus se nutrió de materiales escritos producidos por el propio movimiento (la revista parroquial Compartiendo y los libros de Peries) permitiéndome conocer los modos en que se presenta tanto a los miembros como a potenciales adeptos, y las formas en que establece vínculos con otras esferas sociales. Relevé documentos eclesiales, leyes y decretos municipales, prensa católica y secular. Dada la relevancia de las redes sociales virtuales como herramienta de difusión de la obra de Peries y de socialización de testimonios y experiencias mapeé periódicamente sitios, agencias de noticias, blogs y páginas webs tanto religiosas como seculares.

En lo referido a las entrevistas, los primeros contactos surgieron tanto de mi entorno social e incluso familiar y de relaciones previas a la investigación. A través de los contactos establecidos, como una "bola de nieve", fui ampliando el universo de informantes, de manera que las entrevistas (estructuradas, semiestructuradas, abiertas y la reconstrucción de historias de vida) fueron realizadas a personas de distintos rangos etarios, género y posición socioeconómica; a seguidores y 
participantes activos del movimiento, religiosos, médicos y agentes sanitarios; y en el ámbito cercano a la parroquia, sus hogares, consultorios o puntos acordados. En las narraciones que recibí, en tanto forma distintiva de construir la realidad, los actores situaban sus experiencias dentro de un espacio temporal mayor, reflejaban la participación en mundos sociales y morales específicos $y$, por último, conjugaban recursos personales y culturales ${ }^{(41)}$. Dada la temática de este trabajo, eran recursos para interpretar y actuar sobre los daños a la propia salud o de otros permitiendo atravesar la incertidumbre que rodea la enfermedad y el sufrimiento. Todos mis interlocutores supieron los objetivos de la investigación que desarrollaba y accedieron voluntariamente a participar en ella.

Propongo mostrar que en los eventos de enfermedad se conjugan recursos para lograr la comprensión de aquello que está sucediendo y, luego, actuar para restablecer la salud. Como mis datos provienen de la etnografía antes mencionada, en la gestión de las experiencias que comprometen a su organismo o al de terceros, los actores imbrican principalmente dos recursos: la biomedicina y la cosmología religiosa. De acuerdo a una cronología tipificada de atribución de sentidos, desarrollaré primero el recurso a la medicina científica y, en un segundo momento, a la cosmología religiosa.

\section{Usos de la biomedicina: aciertos y limitaciones}

En las narraciones de mis interlocutores, las primeras caracterizaciones que ofrecen de los cambios negativos percibidos en su experiencia corporal y anímica son amplias y de una precisión que oscila entre la enumeración de síntomas y el autodiagnóstico. Dolores en determinadas partes del cuerpo, tristeza, depresión por estar atravesando situaciones de dolencias físicas o psíquicas propias o de un familiar o amigo, malas relaciones con el entorno familiar, falta de trabajo, dificultades para lograr un embarazo son algunos de los malestares referidos. Lograr una definición más exacta y rigurosa pondrá en evidencia prácticas de apropiación, reinterpretación y combinación de diversos sistemas médicos, siendo la biomedicina el primero de ellos. Esta medicina, al basarse en un discurso objetivo, es la voz autorizada al momento de nombrar y tratar males y padecimientos. Dada su legitimidad sociohistóricamente construida y su eficacia científicamente comprobada, los futuros asistentes a las celebraciones de Peries recurren a ella para comprender los eventos que conciernen a su cuerpo y actuar sobre ellos. Conozcamos entonces de qué maneras este recurso terapéutico opera en las interpretaciones de estos sujetos.

En la consulta con el profesional de la salud, y guiados por este, los pacientes describen sus sensaciones y malestares. Si tal como decía Austin podemos hacer cosas con determinadas palabras expresadas en circunstancias apropiadas $^{(42)}$, el diagnóstico que resulta del encuentro médico, en tanto integrante de un discurso científico y socialmente legítimo, tiene un doble poder performativo. En primer lugar, al etiquetar un conjunto de características, transitorias o permanentes, de una persona como síntomas de una enfermedad que puede ser tratada mediante determinadas prácticas crea el estado "enfermo" (y puede ratificar la etiqueta previamente construida por el actor). Segundo, y estrechamente asociado a la primera acción, convierte a una persona en "paciente" que padece aquello que la etiqueta designa y debe responder a las prescripciones indicadas por el especialista, el médico. De esta manera, el diagnóstico médico será uno de los primeros recursos que, situado en una secuencia mayor, permita suturar sentidos sobre los malestares de los futuros asistentes a las celebraciones de Peries. Más aún, como este discurso es fundador de verdad ${ }^{(43)}$ justificará y legitimará esa asistencia.

Durante mi trabajo de campo, el lenguaje técnico y objetivo del conocimiento y procedimiento médicos era una constante que impregnaba las narrativas de mis interlocutores, quienes me relataban la/s enfermedad/ es que tenían, explicaban posibles etiologías, tratamientos realizados o por realizar, 
y comparaban eficacias. Por ejemplo, Cristina, Alejandra y Rosa, las tres con cáncer de mama, conocían los pormenores de las mastectomías como de las cirugías reconstructivas que atravesaron. Sabían también que una de las medicaciones "aumenta el grosor de las paredes del útero y se puede leer como otra vez, otro foco", creando posibles conexiones entre el cáncer que padecían y el de útero. Los detalles sobre endometriosis, la trombofilia como el tratamiento de heparina y el aprendizaje de la técnica para autoaplicarlo inundaban los relatos de Estefanía, Mariana y María Elena; tres mujeres que, estrenando la treintena de años las dos primeras y abrazando los cuarenta la última, tenían problemas para tener hijos.

En esta instancia, tratamos con la enfermedad entendida como mal funcionamiento o maladaptación de los procesos biológicos y psicofisiológicos en el individuo, aquello que los autores anglófonos designan con el término disease ${ }^{(21)}$. Esta concepción actúa por medio de la objetivación y tiene ciertas implicancias $^{(19)}$. Primero, la enfermedad es una "cosa" localizable en un órgano que puede ser extraído o reparado quirúrgicamente. Segundo, y tal como señalaba, moldea la experiencia íntima del evento al otorgar representaciones tranquilizadoras: es el órgano el que no funciona o lo hace deficientemente, es el virus o el microbio que ingresó al cuerpo. Las enfermedades, si bien están encarnadas en los cuerpos de los enfermos son realidades que tienen poco que ver con su persona. Así, la hiperactividad y falta de concentración del hijo de uno de mis entrevistados eran síntomas de trastorno por déficit de atención con hiperactividad (TDAH) y lo medicaron; "no quedar embarazada" resulta de la endometriosis que debe ser removida del cuerpo.

Dada la eficacia científicamente comprobada y la legitimidad socialmente atribuida a la biomedicina, los actores sociales depositan su confianza en los médicos y en los tratamientos prescriptos. Así, una de mis entrevistadas, paciente oncológica, repetía como un mantra "mi médico y mi psicólogo me dijeron 'vos lo único que tenés que escuchar es a los profesionales que te atienden, porque todos los demás te van a querer ayudar, pero cada organismo actúa de una manera distinta". Esta seguridad operaba también como límite a la escucha de las experiencias de otros. Si bien en un primer momento habían contribuido para la elaboración del autodiagnóstico, luego de la inferencia clínica son percibidas como negativas en tanto generan cuestionamientos al criterio y autoridad del profesional. Esta mujer me contaba que le había puesto el freno a una amiga al decirle "yo te pido que por favor no me digas más nada, porque a mí me lo van a resolver mis médicos, porque así me dijeron".

Durante el desarrollo de mi investigación, identifiqué también diferentes usos del discurso biomédico por parte del sacerdote como del propio movimiento generado en torno a su figura. Respecto al primero, y como mencioné, la capacidad sanadora de Peries es la faceta del "don" más difundida a través de narrativas y soportes varios. Si bien, él resiente discursivamente esta condición al inscribirla en el marco religioso y no en una cualidad individual ${ }^{(44)}$, sus homilías enumeran enfermedades y tratamientos biomédicos. Además, durante las esperas, guiados con una voz en los altoparlantes, los fieles rezan una oración creada por el sacerdote y publicada en uno de sus libros, en la cual pide que Jesús derrame su gracia y amor sobre "nuestros hermanos enfermos, sobre aquellos que sufren cáncer, sida y padecen enfermedades mortales" ${ }^{\prime(45)}$.

A nivel institucional, la Cruzada del Espíritu Santo (la asociación clerical que preside Peries), ante la retracción y ausencia del Estado, tomó para sí las demandas sociales de los vecinos del distrito noroeste de la ciudad. En este sentido, con la parroquia Natividad del Señor como núcleo, la asociación expandió de manera centrífuga sus acciones, creando diversas instituciones: escuelas, comedores, un polideportivo y una cancha de bochas. Asimismo, y en correlato con la popularidad alcanzada por el sacerdote como sanador, junto a la Pastoral de la Salud, construyó dos dispensarios que ofrecen atención de numerosas especialidades 
médicas. Realizó también campañas de detección y prevención de diversas patologías, como cánceres de mama y de útero, VIH y problemas de la vista (cataratas, diabetes y/o glaucoma). Es relevante señalar que la incursión institucional de la Cruzada en distintos ámbitos (educación, deporte, tercera edad y salud), en consonancia con las discusiones acerca del lugar de las religiones en las sociedades contemporáneas, evidencia su trabajo de reorganización permanente para dar respuesta y esperanzas frente a las problemáticas cotidianas ${ }^{(12)}$.

En el plano ritual podemos vislumbrar otro uso del discurso biomédico. Una vez concluida la liturgia, el sacerdote brinda una bendición a todos los presentes y existe también la posibilidad de solicitar un encuentro cara a cara con él. Para ello es necesario completar el formulario "Turno enfermos" en la página web de la parroquia que, entre sus requerimientos, incluye documentar una enfermedad con historia clínica o certificado médico. Este requerimiento trae al primer plano, una vez más, la legitimidad socialmente otorgada a la biomedicina al recubrir con un aura de objetividad las restricciones al acceso a los encuentros personales con Peries, al basarse en evidencia científica. También, muestra el poder irrefutable de la escritura ${ }^{(46)} y$, en particular, de la médica. De acuerdo a la información que recabé, esta medida se impuso para ordenar la masividad de la asistencia y frenar la compra-venta de turnos, negocio que alcanzaba las cuatro cifras en dólares.

Ahora bien, la percepción sintomática de los problemas y afecciones, propia de la biomedicina, considera relevante la porción de información útil para el diagnóstico. Hay otro caudal de experiencia del enfermo que queda excluida: sus emociones, la propia percepción del cuerpo y del proceso que atraviesa; exclusión que es resentida por los pacientes. Emergerá así una narrativa que condensará dos características: por un lado, será crítica de la medicina científica y, por otro, sustentará los motivos para trascenderla y buscar otras opciones terapéuticas.

Las críticas abarcan un amplio espectro de insatisfacciones relacionadas con el encuentro con el profesional de la salud y con los procedimientos. "¿Viste cuando los médicos no te saben explicar?" resumía Cacho, uno de mis entrevistados con cáncer de páncreas, a la falta de interés de su cirujano en franquear la distancia que separa el lenguaje del saber docto del cotidiano. Otro foco de quejas atañe a los valores: el desapego y la insensibilidad adjudicada a una mayoría de los profesionales de la salud en pos del cientificismo y objetividad. "El médico que me operó siempre fue extremadamente parco. Así que con él no contaba mucho como para decir todo lo que sentía", refería Alejandra, mientras que Rosa, si bien reconocía la experticia de los profesionales, señalaba "en el [Hospital] Argerich, los médicos son muy capos... pero son muy médicos, hay algo que no me cierra. Ellos sacan, ponen. Para ellos ¿cuál es el problema? Te corto por acá, te saco por allá y vos te quedas asombrado".

Como consecuencia, se construyen las razones que legitiman la búsqueda de otras alternativas médicas que tomen en consideración la experiencia, emotividad e inferencias subjetivas de los enfermos. Estos, que ya habían elaborado un autodiagnóstico, recurrirán a etiologías subjetivas y simultáneamente sociales $^{(15,19,21)}$ para comprender los procesos que hacen que "se sientan mal" - el término anglófono que lo señala es illness ${ }^{(21)}-$, "mejoren" o "se curen". Por lo tanto, no estamos tratando con personas pasivas sino con actores que generan otras interpretaciones $y$ praxis que critican, se solapan y exceden a las propuestas por la biomedicina. Sobre estas otras respuestas cognitivas y prácticas del evento de enfermedad, y la centralidad del encuentro con Peries, trabajaré en la próxima sección.

\section{Las otras medicinas: cosmología religiosa, ritual y sanación}

No limitarse al recurso biomédico y "abrir el juego" a otras opciones médicas conlleva la modificación en las formas de concebir y actuar sobre los daños a la salud. De esta manera, trascender el anatomismo cosificante 
de la biomedicina, tal como lo caracteriza Laplantine ${ }^{(19)}$, da lugar a una concepción holística del evento de enfermedad en la que lo físico, psíquico, emotivo y la dimensión social como la numinosa no son escindibles y se conjugan como posibles etiologías, el mal en sí mismo o como posibles terapias. En este contexto, al enfermar, se perciben como vulneradas las estructuras de contención (familia, trabajo, etc.) propias de los actores, quienes gozarán de buena salud al tener un cuerpo sano, pero también buenas relaciones con la familia y con la comunidad. Para mis entrevistados el restablecimiento de la salud equivalía a lograr un embarazo tras años de búsqueda incluyendo tratamientos de fertilización fallidos, restituir el equilibrio de sus hogares "destruidos por la enfermedad" o "encontrar las fuerzas y las ganas para salir adelante" luego de la muerte de un familiar o de que un cáncer remitido volviera a activarse. Vemos entonces que el abandono de una noción restringida sobre la salud-enfermedad para abrazar una holística no resulta solo de aquellos criticismos proferidos a la medicina científica, reseñados previamente. Implica también un proceso de medicalización de la experiencia social en el que los problemas y sus posibles soluciones sean concebidos en términos de salud-enfermedad.

Sumado a este macroproceso, es necesario que los actores posean una red social heterogénea que los exponga a una gama amplia de información y valores ${ }^{(20)}$. Estos ámbitos de sociabilidad no solo permitirán conocer otras alternativas médicas, sino que garantizarán su eficacia y legitimidad. Como supo decirme Marta, una jubilada mientras esperaba para recibir la imposición de manos de Peries: "yo quiero que el cura me toque, que me haga bien [...] si otro se curó, ¿por qué yo no?".

Mis propios datos ilustran que, al tener conocimiento de otros recursos terapéuticos, las estrategias de los actores los conjugarán en un mismo evento de enfermedad, evaluándolos y reordenándolos en función de cada situación ${ }^{(5,44,47,48)}$. En sus trayectorias, mis interlocutores combinaron tratamientos oncológicos con reiki y meditación o modificaron sus hábitos alimenticios, adoptando dietas macrobióticas u ovolacteovegetarianas. Señalaron también la relevancia de ciertas lecturas como un acompañamiento necesario para afrontar quimioterapias, tratamientos de fertilización y hacer más soportable la angustia que pueden acarrear. Alejandra priorizó a los "maestros orientales que nos dicen que no tenemos que adherirnos a las cosas, eso de desprendernos". Entre ellos ubica a Peries quien, por medio de sus escritos, señala que "las verdaderas riquezas son las morales y espirituales que radican en el interior de cada uno, y que estas riquezas guiarán el camino hacia una felicidad real"(45,49). Finalmente, refirieron a la participación sostenida en comunidades y grupos religiosos o la asistencia puntual a liturgias y ceremonias de sanación o el encuentro con personas socialmente adscriptas a cualidades excepcionales, como ser el caso del "cura sanador".

Para llegar a Peries serán centrales los testimonios de personas del círculo inmediato del enfermo, de personas desconocidas cuyas palabras circulan en foros y blogs de Internet, como de famosos en los medios de comunicación y "que se curaron o mejoraron" por la intervención eficaz del carisma sanador del sacerdote. Marta llega a Rosario "porque me lo recomendó Carlos, el señor que administra el edificio que viene porque tiene algo en la punta del pulmón, un cáncer"; Estefanía afirma haberse decidido por "los testimonios de la gente" ya que "una semana antes de ir vi en tres noticieros, tres noticias distintas de casos que mujeres que no podían tener hijos, que fueron y quedaron embarazadas". Al respecto, y evidenciando el ordenamiento temporal de los recursos médicos, Cacho enumeró "yo llegué al cura Ignacio después de haber hecho todo, probé con reiki, psicología, flores de Bach, una homeópata que me dijo que no tenía nada". Estos elementos son congruentes también con las tendencias identificadas en el contexto religioso argentino mayor, donde se constata que el $45 \%$ de los argentinos acude a Dios en momentos de sufrimiento y el $14,3 \%$ cuando necesita una ayuda específica ${ }^{(50)}$. 
La posibilidad de leer tanto a la asistencia y participación en grupos religiosos como al contacto con seres poderosos como modalidades de atención a la salud se vincula con la gravitación de las matrices religiosas en las biografías de los actores, en un contexto nacional con una fuerte impronta cultural católica $^{(51)}$. Para el caso que trabajé, una amplia mayoría de quienes buscan restablecer la salud con la intervención divina son católicos de carrera, es decir, personas que tienen relaciones de larga data, y ambivalentes, con el credo bajo el cual fueron criados y educados $^{(52)}$. Por ello, además de las diferentes narrativas que testimonian la eficacia sanadora del sacerdote, será el encuadramiento católico de su figura y el accionar el que legitime la posibilidad de comprenderlas como modalidades de atención a la salud. Estefanía explicaba: "yo creo que [Peries] tiene el poder de sanar, yo creo que viene de dios [...] yo nunca iría a un curandero. Lo tomo porque es de la iglesia católica". Para aquellos que tienen un vínculo quebrado con la institución, el sacerdote se inserta en una red de relaciones complejas entre el cielo y la tierra que implica figuras sagradas ${ }^{(53)}$. Estas relaciones exhiben los mismos rasgos que caracterizan a las que acontecen entre humanos como esperanza, amor, proyecciones, entre otras. El poder taumatúrgico que le atribuyen a Peries, con el componente esperanzador de la cura milagrosa, encuentra su lugar y explicación: "yo no fui a probar, yo tengo fe. Para mí no sería raro que alguien pueda ser transmisor de cura, o hacer milagros", relataba Rosa convencida y disipando cualquier duda sobre la eficacia de la sanación.

¿A través de qué prácticas el sacerdote cura? Principalmente, por medio de la "imposición de manos", instancia ritual colmada de efusiones emocionales y corporales. Durante mi trabajo de campo, observé numerosas celebraciones en las que el propio Peries, micrófono en mano y al pie del altar, explicaba la prioridad para recibir la "bendición": "un servidor entregará números para aquellos afligidos graves, para que no se me escapen. A los demás, atenderé rápido y sin detenerme". Un elemento llamativo es la elección de la palabra "atender" para referirse a la imposición de manos. Si como vimos, la percepción de los problemas se realizaba en términos de salud-enfermedad, la búsqueda de respuesta también reflejará el proceso de medicalización. En este sentido, inclinarse por ese vocablo permitiría a los asistentes tipificar la situación de la imposición de manos dentro de una práctica conocida, como ser la relación médico-paciente, en la que existe una distribución desigual del conocimiento sobre el proceso salud-enfermad-atención ${ }^{(4)}$. A mi entender, la relevancia de esta opción no se limita únicamente a favorecer el proceso tipificador. Entre sus acepciones, atender significa "aplicar voluntariamente el entendimiento a un objeto espiritual o sensible; tener en cuenta o en consideración algo; mirar por alguien o algo, o cuidar de él o de ello"(54). Por lo tanto, la atención que Peries dispensa a los asistentes da cuenta del establecimiento de un tipo particular de relación entre los sujetos de la interacción, caracterizada por una mediación sensible y emotiva colmada de abrazos, caricias, palabras al oído, llantos y risas que validan y retroalimentan el holismo de las nociones de salud-enfermedadatención. El sacerdote, diferenciándose de los profesionales inscriptos en la medicina científica, atiende el mundo anímico de su interlocutor y podrá generar efectos reales en él. Un amplio rango de acciones prueba su intervención eficaz: lograr embarazos, encontrar trabajo, resolver problemas familiares, lograr calma y tranquilidad luego de un período de angustia. Asimismo, la variable emotiva y sensible está presente en las etiologías del sacerdote quien, mixturando las medicinas folks y la psicología, asocia el trastorno generalizado del desarrollo (TGD) a un susto o episodio traumático durante el embarazo.

Por otro lado, la medicalización de la experiencia social es fortalecida discursivamente. En una de las pocas entrevistas brindadas a un medio de circulación nacional afirmó que "el ser humano sufre mentalmente, psíquicamente, emocionalmente y también hay muchos problemas familiares que producen dolor"(55). En las liturgias, 
refiere a "los males que aquejan" la vida cotidiana como ser la falta de seguridad social, laboral, económica y la violencia que, como resultado de dichas carencias, imperan en la sociedad.

Vemos así que el abandono de la noción restringida de enfermedad para dar paso a una global viabiliza el encuentro con el cura sanador como una modalidad posible de atención de los daños a la salud. Sin embargo, este pasaje no anula a la medicina científica como respuesta práctica, sino que concentra sus esfuerzos en identificar profesionales que, inscriptos en ella, presenten características singulares: deben estar atentos a los elementos a partir de los cuales los pacientes significan sus vidas y construyen su forma de pensar y ser capaces de escuchar sus miedos y preocupaciones. Más aún, las narrativas de mis interlocutores destacaban aquellos profesionales que, entre las recomendaciones, incluían una visita al "cura sanador" ya que habían comprobado su influencia positiva entre quienes, por ejemplo, enfrentaban inconvenientes para tener hijos. Desde el punto de vista de los asistentes a las misas de Peries, esta sugerencia da cuenta de dos elementos evaluados positivamente. Primero, que dentro de los procesos de salud-enfermedad-atención tiene relevancia la esfera espiritual-emocional. Segundo, y retomando la legitimidad del discurso biomédico analizada en la sección anterior, comprueban "científicamente" que asistir a las celebraciones en la parroquia rosarina es eficaz para el restablecimiento de la salud. En términos analíticos, estábamos tratando con procesos de combinación terapéutica propiciados por los médicos.

El lugar que los profesionales de la salud otorgan a las terapias no biomédicas presenta una amplio rango de posibilidades que abarca la censura y la penalización hasta la derivación del paciente a otro contexto terapéutico, e incluye posiciones intermedias como la aceptación pasiva o la apropiación de otras formas médicas o terapéuticas ${ }^{(7)}$. Para el caso que analizo, encuentro tendencias inclusivas que atestiguan, además, la heterogeneidad del mundo biomédico en la actualidad local.
Dentro del universo de los doctores, una de las entrevistas que Ilamó especialmente mi atención fue la de un médico clínico recibido en la Universidad de Buenos Aires, especializado en psicoinmunoendocrinología y acupuntor. Además de una profusa actividad docente, al momento de nuestros encuentros, trabajaba en los servicios de dolor y de cuidados paliativos de personas con cáncer de un hospital del sector público de la Ciudad Autónoma de Buenos Aires y atendía también en dos consultorios privados. En nuestras charlas, tranquila y pausadamente, fue desarrollando las nociones que guían su práctica profesional: "los cambios emocionales, los nervios, el estrés influyen de forma negativa en el cuerpo que refleja todo lo que nos pasa. La acupuntura ayuda a restituir la armonía perdida". En más de una oportunidad recibió pacientes oncológicos y mujeres con problemas de fertilidad que habían asistido a las misas de Peries logrando resultados evaluados positivamente por los pacientes. Sobre ello se pronunció diciendo "no sorprende, porque la fe en poder mejorar es vital en este tipo de enfermedades".

La influencia del orden anímico aparece también reflejada en la perspectiva de una de las ginecólogas que entrevisté. Llegué a esta especialista en trombofilia y abortos recurrentes porque las fieles la referían constantemente durante las largas esperas previas al ingreso a la parroquia rosarina. Constaté luego que su nombre colma los blogs sobre infertilidad. A sabiendas del uso de Internet como fuente de información, esta doctora desaconseja a sus pacientes la lectura de blogs, perfiles de Facebook y páginas webs con testimonios sobre fertilidad "porque no quiero que estén buscando un bebé pensado en que pueden perderlo porque leyeron que alguien lo perdió".

En el paradigma de estos profesionales, como en el movimiento religioso, la dupla normal-patológico es suplantada por armonía-desarmonía, equilibrio-desequilibrio. Si, como vimos, para la medicina científica las enfermedades son "cosas" aislables y destruibles, aquí tratamos con desarreglos que deben comprenderse en su globalidad. Estar 
enfermo implica la ruptura del equilibrio del hombre con su propia persona, el orden social y/o el $\operatorname{cosmos}^{(19)}$. Por ello, será relevante el orden numinoso, aquello que escapa a la razón y control humanos, al fundarse en el ámbito espiritual de la vida como factor explicativo de la eficacia o no de un tratamiento. Como señalé en otro trabajo(5), esta continuidad alcanzará su máxima expresión en la sanación, en la cual el proceso de salud-enfermedad-atención incluye un nivel espiritual que integra la experiencia cotidiana y el sufrimiento se extiende desde lo anímico y físico a un contexto más amplio. De esta manera, las soluciones a los problemas serán más sorprendentes y la intervención numinosa más evidente, cuando la evaluación biomédica concluye en la presencia de trastornos severos o de una enfermedad con bajas tasas de supervivencia o que implica procedimientos invasivos y prolongados. Vale recordar que al sacerdote Peries se lo reconoce como "especialista" en la cura de enfermedades como cáncer y la resolución de problemas asociados a la infertilidad o al TGD.

En suma, el carácter holista del proceso salud-enfermedad-atención parte de la unidad de los fenómenos físicos y espirituales, racionales y emocionales, en detrimento de una división estanca entre cuerpo y alma. Ante las insuficiencias de la biomedicina para abordar la esfera espiritual-sensorial, estas personas buscaron alternativas médicas y religiosas en las que dicha dimensión sea relevante. En este punto, el contacto con el cura sanador y la participación en los rituales de sanción se perciben como una parada, e incluso como destino final de un recorrido terapéutico que permitirá proyectar una realidad futura y alternativa al presente enfermo de un individuo, su familia y/o círculo de sociabilidad inmediato.

\section{CONCLUSIONES}

En este trabajo me propuse explorar las vinculaciones entre salud y terapéuticas religiosas basándome en mi investigación etnográfica en un movimiento católico de sanación. Particularmente, me centré en las modalidades en que los actores configuran las respuestas cognitivas y prácticas que los eventos de enfermedad requeridos para su gestión.

La primera parte del artículo estuvo dirigida a mostrar algunos abordajes de las ciencias sociales sobre el proceso de saludenfermedad-atención, puntualizando la pluralidad jerarquizada de sistemas médicos y los procesos de combinación efectuados por los actores. Seguidamente, presenté la base empírica de mi investigación y la metodología de construcción y recolección de los datos. Luego, describí y analicé las trayectorias y estrategias desplegadas por los actores en pos del restablecimiento de la salud poniendo en foco las concepciones, prácticas y experiencias en torno a la salud-enfermedad-atención.

Vimos entonces que un evento disruptivo de la experiencia corporal o anímica es percibido como el origen de un amplio espectro de malestares definidos con mayor o menor precisión. Se plantea entonces la necesidad de gestionar dicha disrupción para comprenderla y solucionarla. Advertimos así que los actores combinan recursos terapéuticos diversos, reconociéndoles aciertos, falencias y eficacias específicas. Entre quienes realicé mi investigación, la biomedicina y las terapias religiosas constituyeron los recursos centrales. Asimismo, cada uno fue referido en momentos específicos de las trayectorias generando una cronología de atribución de sentidos.

El primer recurso movilizado para obtener una respuesta cognitiva sobre el evento de enfermedad es la biomedicina. Esta otorga un diagnóstico basado en el conocimiento técnico y científico y ofrece representaciones tranquilizadoras que moldean la experiencia de estos eventos. Señalé también que el movimiento de Peries la refiere en el discurso individual del sacerdote como en las prácticas institucionales para fundamentar algunas de las acciones emprendidas.

Existe también una narrativa crítica de la medicina científica que abarca un amplio rango de quejas pero que, para el caso que presenté, puede sintetizarse en la incapacidad 
para abordar la esfera espiritual-sensorial de los enfermos. En consecuencia, se torna necesario apelar, en un segundo momento, a otros recursos en los que dicha dimensión sea considerada fuente legítima de indagación, explicación y acción de los padecimientos. Cobrará así importancia la terapia religiosa como recurso interpretativo y práctico para los daños a la salud.

El pasaje de un recurso al otro conlleva una modificación en la conceptualización de los eventos disruptivos. Se abandona una noción restringida a procesos biológicos y psicofisiológicos normales-patológicos para dar paso a una global entendida en términos equilibrio-desequilibrio de las múltiples dimensiones de la experiencia vital: física, psíquica, emotiva, social y numinosa no son escindibles, sino que construyen un continuum en el que los actores tejen activamente sus interpretaciones y prácticas. Asimismo, advertimos que las respuestas cognitivas y prácticas se imbrican mutuamente ya que solo una noción amplia de la enfermedad puede incluir una dimensión ritual-religiosa como forma de atención de la salud.

En la presentación del caso mencionaba que lo refería como movimiento en tanto esfuerzo colectivo por modificar modos instituidos de hacer, específicamente modos de gestionar la salud. Estas novedades, entramadas en un doble marco institucional (la iglesia católica y la asociación clerical dirigida por el sacerdote), instalan también la posibilidad de repensar las articulaciones actuales entre dos dominios que gozan de legitimación social y simbólica en la sociedad argentina: el catolicismo y la biomedicina. Los datos presentados señalan que ante las certezas que provee la medicina científica, se erige el componente esperanzador de la terapia religiosa dando lugar a una asociación complementaria y competitiva (en lo que respecta a eficacias diferenciales socialmente atribuidas) entre la biomedicina y el carisma sanador. Más aún, la institución regida por Peries identifica dicha asociación y acciona desde ella al proponer un modo de gestión de la salud basado en una "alianza de medicinas" a partir de la aplicación, tal como vimos, de diversos dispositivos que enlazan elementos de cada una de ellas como ser la clasificación y jerarquización de enfermedades, el otorgamiento de turnos de atención basados en evidencia biomédica y ciertos elementos rituales específicos. Finalmente, la propia figura del sacerdote sintetiza esta alianza al operar como un mediador que reconoce la racionalidad biomédica y valoriza la fe religiosa en el proceso de curación.

En suma, el movimiento de sanación formado en torno a la figura de Ignacio Peries fue un terreno fértil para explorar varias problemáticas imbricadas, como ser la pluralidad y combinación de sistemas médicos, la medicalización de la experiencia social, las eficacias disputadas de los recursos terapéuticos y la vigencia del recurso a lo sagrado en las sociedades contemporáneas. Hemos conocido, entonces, una de las modalidades de gestión simbólico-práctica de la salud, la enfermedad y el sufrimiento que se dirime en los intersticios entre salud y religión.

\section{AGRADECIMIENTOS}

El presente trabajo integra una investigación mayor financiada con dos becas del Consejo Nacional de Investigaciones Científicas y Técnicas. La primera de ellas fue obtenida con el proyecto Nuevas experiencias de lo religioso en un catolicismo sin católicos: Identidad, ritual y sanación en el movi- miento carismático del Padre Ignacio realizada en el período 2011-2014, mientras que, la segunda, por el trabajo Imaginarios católicos: nuevas identidades y pertenencias en el catolicismo argentino contemporáneo, abarcó los años 2014-2016. Agradezco a los evaluadores anónimos de este trabajo que con su lectura atenta aportaron solidez en su argumentación. 


\section{REFERENCIAS BIBLIOGRÁFICAS}

1. Evans-Pritchard EE. Brujería, magia y oráculos entre los Azande. Barcelona: Anagrama; 1976.

2. Lévi-Strauss C. La eficacia simbólica. In: LéviStrauss C. Antropología estructural. Barcelona: Paidos; 1987. p. 211-227.

3. Turner V. La selva de los símbolos: aspectos del ritual ndembu. Madrid: Siglo XXI Editores; 1999.

4. Carozzi MJ. Consultando a una Mãe de Santo: un análisis de la construcción social del efecto mágico. Revista de Investigaciones Folklóricas. 1993;8:68-79.

5. Olmos Álvarez AL. "Venid a mí todos los afligidos": salud, enfermedad y rituales de sanación en el movimiento católico carismático del Padre Ignacio. Ciencias Sociales y Religión/Ciências Sociais e Religião. 2015;17(22):52-70.

6. Puglisi R. Alimento para el cuerpo y el espíritu: prácticas alimentarias y cantos rituales en los grupos Sai Baba argentinos. Revista Cultura y Religión. 2014;8(2):129-147.

7. Saizar MM. De la censura a la complementariedad domesticada: reflexiones en torno a los modos de vinculación de la biomedicina con otras medicinas. In: Saizar MM, Krmpotic C, editoras. Políticas socio-sanitarias y alternativas terapéuticas: intersecciones bajo la lupa. Buenos Aires: Espacio Editorial; 2016. p. 25-42.

8. Giménez Béliveau V. Terapéuticas católicas, males modernos: procesos de sanación y exorcismo en Argentina. Sociedad y Religión. 2017;27(47): 33-59.

9. Idoyaga Molina A. Reflexiones sobre la clasificación de medicinas: análisis de una propuesta conceptual. Scripta Ethnologica. 2005;27:111147.

10. Bindi S. L'événement de la maladie entre pratiques et structures: analyse dialogique d'un épisode de maladie en Uttarakhand. Mondes contemporains. 2012;(1):13-33.

11. Esquivel JC. Catolicismo y modernidad en Argentina: ¿de la confrontación a la conciliación? Estudos de Religião. 2013;27(2):193-213.

12. Hervieu-Léger D. La religion pour mémoire. Paris: Le Cerf; 1993.

13. Ludueña GA. Localidad, modernidad y performance misional en la migración de religiosos católicos a la Argentina a comienzos del siglo
XX. Relaciones: Estudios de Historia y Sociedad. 2010;31(124):91-121.

14. Olmos Álvarez AL. Construyendo carismas en el catolicismo contemporáneo argentino: un estudio etnográfico sobre el movimiento en torno al Padre Ignacio Peries. [Tesis de doctorado]. Buenos Aires: Universidad Nacional de San Martin; 2016.

15. Garro LC. Hallowell's challenge: explanations of illness and cross-cultural research. Anthropological Theory. 2002;2(1):77-97.

16. Ceriani Cernadas C. El poder de los símbolos: magia, enfermedad y acto médico. Revista del Hospital Italiano de Buenos Aires. 2006;26(3):87-93.

17. Comaroff J. Body of power, spirit of resistance: the culture and history of a South African people. Chicago: Chicago University Press; 1985.

18. Good C. Ethnomedical systems in Africa. New York: The Guilford Press; 1987.

19. Laplantine F. Antropología de la enfermedad: estudio etnológico de los sistemas de representaciones etiológicas y terapéuticas en la sociedad occidental contemporánea. Buenos Aires: Ediciones del Sol; 1999.

20. Siahpush M. A critical review of the sociology of alternative medicine: research on users, practitioners and the orthodoxy. Health. 1999;4(2):159178.

21. Kleinman A, Eisenberg L, Good B. Culture, illness, and care: clinical lessons from anthropologic and cross-cultural research. Annals of Internal Medicine. 1978;88(2):251-258.

22. Menéndez E. La enfermedad y la curación: ¿qué es medicina tradicional? Alteridades. 1994;4 (7):71-83

23. Menéndez EL. Modelo hegemónico, modelo alternativo subordinado, modelo de autoatención; caracteres estructurales. In: Hacia una práctica médica alternativa: hegemonía y auto atención (gestión) en salud. México: Centro de Investigaciones y Estudios Superiores en Antropología Social; 1983.

24. Maués RH. "Bailando com o Senhor": técnicas corporais de culto e louvor (o êxtase e o transe como técnicas corporais). Revista de Antropologia. 2003;46(1):10-40.

25. Maués RH. Catolicismo e xamanismo: comparação entre a cura no movimento carismático e na pajelança rural amazônica. Ilha Revista de Antropologia. 2002;4(2):51-77. 
26. Machado MDC. Carismáticos e Pentecostais: adesao religiosa na esfera familiar. Campinas: Ed. Autores Associados/ANPOCS; 1996.

27. Cabrera P. Nuevas prácticas, nuevas percepciones: la experiência de la Renovación Carismática Católica. Ilha Revista de Antropologia. 2001;3(1):121-137.

28. Chesnut RA. A preferential option for the spirit: the Catholic charismatic renewal in Latin America's new religious economy. Latin American Politics and Society. 2003;45(1):55-85.

29. Steil CA. Catholic Charismatic Renewal: ia way into or out of catholicism?; ethnography of the São José group, in the city of Porto Alegre, Brazil. Religião \& Sociedade. 2004;24(1):182-190.

30. Silveira EJS. A "posse do Espírito": cuidado em si e salvação; uma análise do imaginário da Renovação Carismática Católica. Rhema. 2000;6(23):143-169.

31. Prandi R. Um sopro do espiritu: a renovação conservadora do catolicismo carismático. São Paulo: EDUSP; 1997.

32. Silveira EJS. Pluralidade Católica: um esboço de novos e antigos estilos de crença e pertencimento. Sacrilegens. 2004;1(1):153-174.

33. Várguez Pasos LA. El movimiento de Renovación Carismática en el Espíritu Santo y el Magisterio de la Iglesia Católica. De la sospecha a la aceptación. Sociedad y Religión: Sociología, Antropología e Historia de la Religión en el Cono Sur. 2008; XX(30-31):7-30.

34. Várguez Pasos LA. Construyendo y reconstruyendo las fronteras de la tradición y la modernidad: la iglesia católica y el Movimiento de Renovación Carismática en el Espíritu Santo. Convergencia Revista de Ciencias Sociales. 2008;(46):195-224.

35. Renold JM. El Padre Ignacio, sanación y eficacia simbólica. In: Renold JM, Dezorzi S. Miradas antropológicas sobre la vida religiosa: el padre Ignacio, sanación y eficacia simbólica y otros ensayos. Buenos Aires: CICCUS; 2008. p. 125-147.

36. Olmos Álvarez AL. De la conveniencia a la convivencia. Explorando los límites del carisma en el catolicismo contemporáneo argentino. Journal of the Sociology and Theory of Religion. 2017; 5:51-75.

37. Salinas L. Reformulaciones de la religión católica: el padre Ignacio y la vigencia de la hegemonía católica en Rosario. [Tesina de licenciatura]. Rosario: Universidad Nacional de Rosario; 2007.
38. Carozzi MJ. El concepto de marco interpretativo en el estudio de movimientos religiosos. Sociedad y Religión. 1998;(16-17):33-52.

39. Ricoeur P. Introducción a la simbólica del mal. Buenos Aires: Megápolis; 1976.

40. Guber R. El salvaje metropolitano: reconstrucción del conocimiento social en el trabajo de campo. Buenos Aires: Paidós; 2004.

41. Garro LC. Narrating troubling experiences. Transcultural Psychiatry. 2003;40(1):5-43.

42. Austin JL, Urmson JO. Cómo hacer cosas con palabras: palabras y acciones. Barcelona: Paidós; 1990.

43. Foucault M. El nacimiento de la clínica. Una arqueología de la mirada médica. Buenos Aires: Siglo XXI Editores; 2004.

44. Olmos Álvarez AL. "Soy un instrumento de Dios": un análisis etnográfico del carisma en el catolicismo contemporáneo. Tabula Rasa. 2015;(23):289-311.

45. Peries I. Reflexiones de vida. Rosario: Tinta Roja; 2002.

46. Ong WJ. Oralidad y escritura: tecnologías de la palabra. Buenos Aires: Fondo de Cultura Económica; 1996.

47. Olmos Álvarez AL. "Los caminos del Señor": aproximaciones al estudio de las trayectorias católicas carismáticas en Argentina. Sociedad y religión. 2013;XXIII(40):143-179.

48. Olmos Álvarez AL, Irrazábal G. Catholicisme et Santé: les itinéraires de guérison biomédicale et spirituelle dans I'Argentine contemporaine. Taller Usages, pratiques et régulations político-religieuses sur le corps: débats et perspectives dans le terrain des sciences sociales; Paris; 2016.

49. Peries I. Les doy mi paz. Rosario: Tinta Roja; 2006.

50. Mallimaci F, Esquivel JC, Irrazábal G. Primera encuesta sobre creencias y actitudes religiosas en Argentina [Internet]. Buenos Aires: CEIL, PIETTE, CONICET; 2008 [citado 3 feb 2015]. Disponible en: https://tinyurl.com/y88y8j5z.

51. Mallimaci F. Atlas de las creencias religiosas en la Argentina. Buenos Aires: Biblos; 2013.

52. Olmos Álvarez AL. Otro catolicismo posible: institución, dios y agentes católicos en las experiencias biográficas de las fieles. Cultura y Religión. 2017;XI(1):4-22. 
53. Orsi RA. Between heaven and earth: the religious worlds people make and the scholars who study them. Woodstock: Princeton University Press; 2005.

54. Real Academia Española. Diccionario de la lengua española [Internet]. 30a ed. Madrid: Real Academia Española; 2016 [citado 23 mar 2016]. Disponible en: http://dle.rae.es/?id = 4Ce1uOL.
55. Favarel ML. "Yo sé que tengo un don especial de Dios que no puedo explicar": entrevista al padre Ignacio [Internet]. La Capital; 2012 [citado 31 oct 2012]. Disponible en: https://tinyurl.com/ yc4e3mln.

\section{FORMA DE CITAR}

Olmos Álvarez AL. Entre médicos y sanadores: gestionando sentidos y prácticas sobre el proceso de salud-enfermedad-atención en un movimiento carismático católico argentino. Salud Colectiva. 2018;14(2):225-240. doi: 10.18294/ sc. 2018.1530.

Recibido: 14 de julio de 2017 | Versión final: 2 de diciembre de 2017 | Aprobado: 28 de diciembre de 2017

utilizada con finalidades comerciales, a menos que se obtenga el permiso. 